\title{
Necrotic Uveal Melanoma Mimics Orbital Cellulitis: A Review
}

\author{
Ahmad Abdel-Aty ${ }^{a}$ Wendy L. Linderman $^{b}$ Ninani Kombo ${ }^{b}$ John Sinard ${ }^{b, c}$ \\ Renelle Pointdujour-Lim ${ }^{b}$

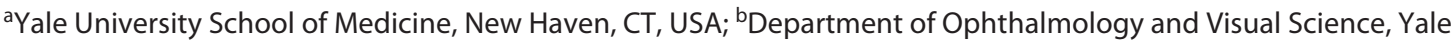 \\ University School of Medicine, New Haven, CT, USA; 'Department of Pathology, Yale University School of Medicine, \\ New Haven, CT, USA
}

\section{Keywords}

Necrotic uveal melanoma · Orbital cellulitis · Orbital

inflammation · Spontaneous necrosis

\begin{abstract}
Background: Uveal melanoma is the most common primary intraocular malignancy in adults, often resulting in painless vision loss. We report a case of necrotic uveal melanoma presenting with orbital inflammation mimicking orbital cellulitis and present a comprehensive review of the literature and tabulation of reported cases. Summary: Our review found 44 published reports of spontaneously necrotic uveal melanoma involving 55 patients. Of these reports, 26 patients (47\%) presented with orbital cellulitis. Presenting symptoms of necrotic uveal melanoma with orbital cellulitis included proptosis (82.8\%), pain (80.7\%), vision loss (61.5\%), and restricted extraocular movements (46.2\%). Key Messages: Uveal melanoma can rarely mimic orbital cellulitis. Autoinfarction and tumor necrosis causes secondary orbital inflammation. Intraocular malignancy must remain in the differential for patients with orbital inflammation and vision loss.
\end{abstract}

(c) 2021 S. Karger AG, Basel
(C) 2021 S. Karger AG, Basel

www.karger.com/oop

\section{Introduction}

Uveal melanoma is the most common primary intraocular malignancy, accounting for $85 \%$ of intraocular malignancies and affecting $2-8$ people per million population $[1,2]$. Uveal melanoma develops from uveal melanocytes of neural crest origin, most commonly from the choroid or ciliary body (98\%) [1-3]. Patients have varying presentations with blurry vision being the most commonly reported symptom (37.8\%), followed by an asymptomatic presentation in $30.2 \%$, photopsia in $8.6 \%$, floaters in $7 \%$, visual field loss in $6.1 \%$, and pain in $2.4 \%$ [4].

Orbital inflammation can be a rare manifestation of uveal melanoma. We report a patient with necrotic uveal melanoma presenting with acute painful vision loss, proptosis, and orbital inflammation. In addition, we detail a review of published cases of necrotic uveal melanoma presenting with orbital cellulitis.

\section{Case Presentation}

A 64-year-old Caucasian male presented to the Emergency Department with a 3-day history of acute vision loss of the right eye with associated periorbital edema, pain radiating to the forehead, and subjective fever and chills. He had no past medical history. On

\footnotetext{
Karger $\stackrel{2}{\circ}$
}

Correspondence to:

Renelle Pointdujour-Lim, renelle.lim@yale.edu 

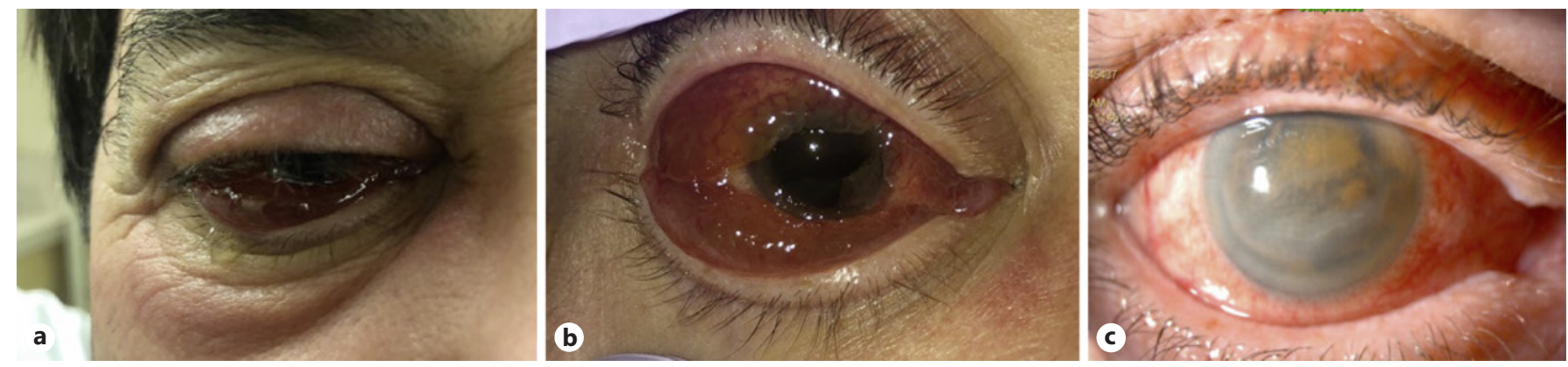

Fig. 1. a, b External photographs of the right eye demonstrating eyelid edema and erythema, conjunctival injection and chemosis, and hyphema at initial presentation. c External photograph two months after initial presentation demonstrating conjunctival injection, shallow anterior chamber, and fibrinoid debris in the anterior chamber without a clear view of the pupil.

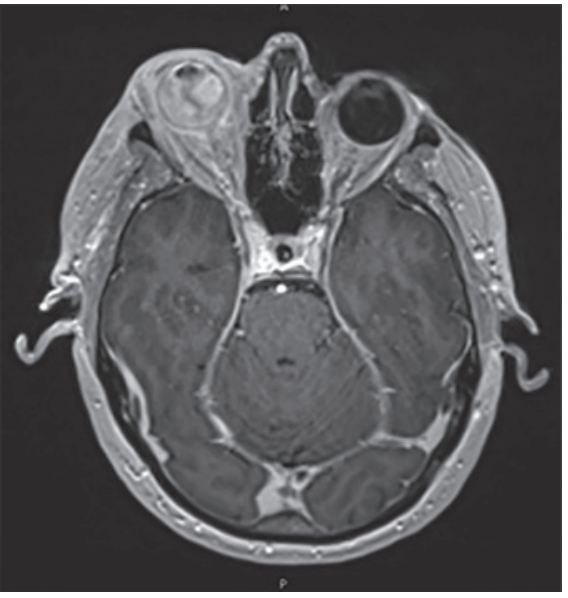

Fig. 2. Axial T1 MRI with contrast image demonstrating right eye proptosis, orbital inflammation, intraocular debris and hemorrhage, and an enhancing intraocular mass.

examination, he was afebrile. Visual acuity was no light perception in the right eye and 20/20 in the left eye. A right relative afferent pupillary defect was present. There was mild right-sided proptosis. The intraocular pressure of the right eye was elevated to $88 \mathrm{~mm} \mathrm{Hg}$. The right upper and lower eyelids were tender to palpation, edematous, and erythematous (shown in Fig. 1a). Anterior segment examination demonstrated diffuse severe conjunctival chemosis and a total hyphema obscuring visualization of the posterior segment (shown in Fig. 1b, c). B-scan ultrasonography demonstrated vitreous hemorrhage but no retinal detachment or visible mass. The left eye was unremarkable. MRI of the orbits demonstrated proptosis and diffuse orbital inflammation; however, there was no evidence of sinusitis, foreign body, or abscess. There was enhancement of an intraocular lesion in the right eye (shown in Fig. 2). Though the imaging was suspicious for malignancy, an intraocular tumor could not be definitively distinguished from intravitreal hemorrhage. There was no improvement in symptoms despite intravenous vancomycin and ceftriaxone. Laboratory testing, including a complete blood count, coagulation panel, hypercoagulability panel, and sickle cell screen, was all within normal limits. Magnetic resonance venogram was negative for thrombosis or fistula. Though definitive diagnosis could not be made by imaging, the patient was clinically diagnosed with an intraocular tumor. Enucleation of the blind and painful eye was performed. Histopathology demonstrated a pigmented mass of necrotic spindled and epithelioid cells in the posterior segment (shown in Fig. 3a-c). There was corneal stromal neovascularization and loss of endothelial cells. The iris, ciliary body, and neurosensory retina were necrotic. Due to the extent of the necrosis, the presence of iris neovascularization and angle closure could not be excluded. The choroid was still viable and showed a reactive inflammatory process to the necrotic mass. There was no viable tumor or extrascleral extension. The sclera was edematous. Immunohistochemistry staining with Sox 10 was unsuccessful as the necrotic cells did not stain. However, Fontana-Masson staining confirmed that the pigment present within the necrotic cells and within the macrophages was melanin. Systemic surveillance with MRI of the abdomen and pelvis demonstrated a $0.9-\mathrm{cm}$ enhancing right hepatic lobe mass. Follow-up imaging demonstrated additional hepatic metastases. Core needle biopsy of the hepatic mass stained positive for Melan-A and SOX10, consistent with metastatic uveal melanoma. The patient was treated with immune checkpoint inhibitors, nivolumab $1 \mathrm{mg} /$ $\mathrm{kg}$ and ipilimumab $3 \mathrm{mg} / \mathrm{kg}$, which was followed by microwave ablation of the hepatic lesions. Subsequent surveillance imaging of the chest, abdomen, and pelvis demonstrated progression of metastatic lesions in all segments of the liver and multiple lung nodules of indeterminate significance. Surveillance imaging of the brain remained negative. The patient is alive at 17 months from the initial presentation, 13 months after enucleation.

\section{Literature Review}

A comprehensive review of the relevant literature with a tabulation of previously reported cases of necrotic uveal melanoma presenting as orbital cellulitis was conducted (Table 1). Scopus and PubMed were searched for cases of spontaneous necrotic uveal melanoma that presented with 

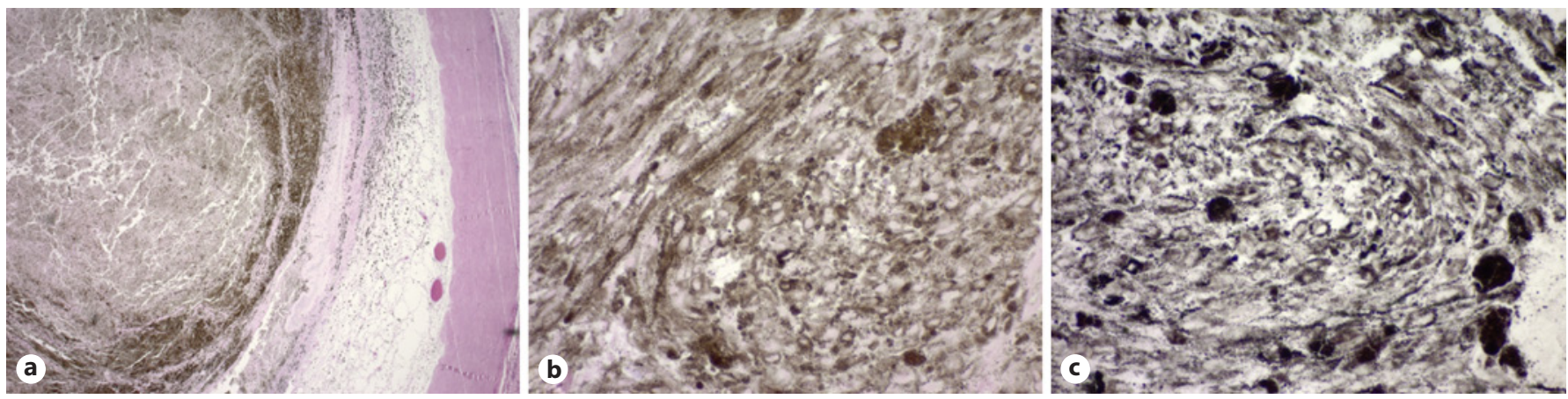

Fig. 3. $\mathbf{a} \times 2$ magnification micrograph of the posterior chamber of the globe. From left to right: necrotic tumor, melanin-laden macrophages (melanophages), edematous choroid, and sclera (hematoxylin and eosin stain). b $\times 60$ magnification micrograph of tumor showing "ghosts" of both spindled and epithelioid cells. Pigment is present in the majority of these cells (hematoxylin and eosin stain). $\mathbf{c} \times 60$ magnification micrograph of FontanaMasson stain confirming that the pigment in the cells is melanin.

orbital inflammation. Cases were included if they presented with orbital inflammation and symptoms of orbital cellulitis including proptosis and restriction of extraocular movements, or if they were described as presenting with "orbital cellulitis" or "pseudocellulitis," and if they were later diagnosed as necrotic uveal melanoma by histopathology. Cases were excluded if symptoms presented after treatment or if there was no histopathologic confirmation of diagnosis.

Our review found 44 published reports and series of spontaneously necrotic uveal melanoma involving 55 patients. Of the 55 patients with necrotic uveal melanoma, 26 patients $(47 \%)$ presented with orbital inflammation. Cases 1-26 are shown in Table 1. The mean age of presentation was 64 years (range $24-89$ years), and $69.2 \%$ of patients were male. Presenting symptoms included proptosis $(82.8 \%)$, pain $(80.7 \%)$, vision loss $(61.5 \%)$, and restricted extraocular movements (46.2\%). Of those presenting with vision loss, 50\% presented with chronic ( $>1$ month) gradual visual loss. The fundoscopic view to the choroid was obscured in $58 \%$ of cases due to hyphema, corneal edema, intravitreal hemorrhage, or a dense cataract. Nineteen patients $(73.1 \%)$ received a B-scan ultrasound, 16 patients $(61.5 \%)$ received a CT scan, and 4 (15.3\%) received an MRI. Eighteen (94.7\%) of the B-scan ultrasounds, all of the CT scans, and 3 (75\%) of the MRIs demonstrated evidence of a mass. The uveal melanoma involved the choroid in $80.8 \%$, ciliary body in $7.7 \%$, and was unspecified in $11.5 \%$ of cases. Histologically, $42.3 \%$ had a mixed epithelioid and spindle cell morphology, $23.1 \%$ had a spindle cell morphology, $11.5 \%$ had an epithelioid morphology, and $23 \%$ had an unknown or unreported morphology.

Enucleation was performed in 19 patients (73.1\%), exenteration in 5 patients (19.2\%), and evisceration in 2 pa- tients (7.7\%). All 5 patients who were managed by exenteration had extraocular extension of the uveal melanoma. While there were 2 reported cases, evisceration is usually avoided in patients with a vague clinical history and without adequate visualization of the fundus in an effort to decrease tumor seeding. Of the 2 cases reported by Eagle et al. [5] who underwent evisceration, 1 patient had subsequent surgery to remove the scleral shell; however, there was no evidence of residual tumor (case 1). The second patient (case 19) was found to have extrascleral extension; however, there was no follow-up data regarding further management or outcome. Extraocular extension was present in 10 patients (38.4\%). Of these patients, treatment included exenteration in 5 patients (cases $\# 3,9,13,15$, and 17), enucleation with lateral orbitotomy in 2 patients (cases 11 and 14), enucleation with posterior tenonectomy in 1 patient (case \#24), standard enucleation in 1 patient (case\# 25), and evisceration in 1 patient (case \#19). Two patients with extraocular extension received adjuvant external beam radiation therapy (cases \# 11 and 13). Only 2 patients were reported to have metastatic disease and both received adjuvant treatment consisting of cytarabine (case \#3) and immune checkpoint inhibitors, nivolumab and ipilimumab (case \#26). Three other patients were deceased within 1 year, indicating possible metastatic disease. Only 16 reports (61\%) included follow-up information.

\section{Discussion}

The first published case of necrotic uveal melanoma presenting with orbital inflammation was by Kline et al. [6] in 1977. Since then, there have been an additional 22 


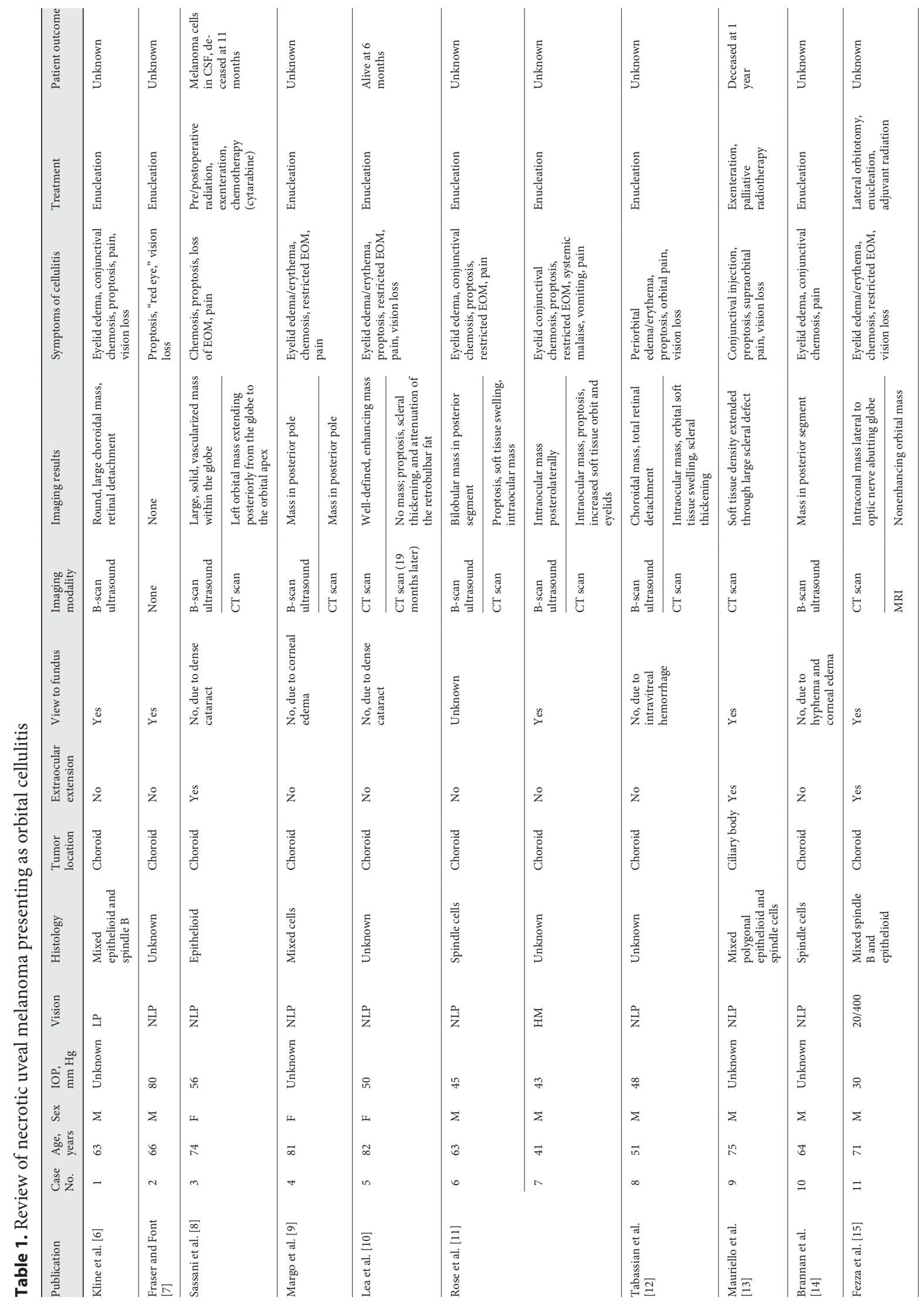

${ }^{4}$ 


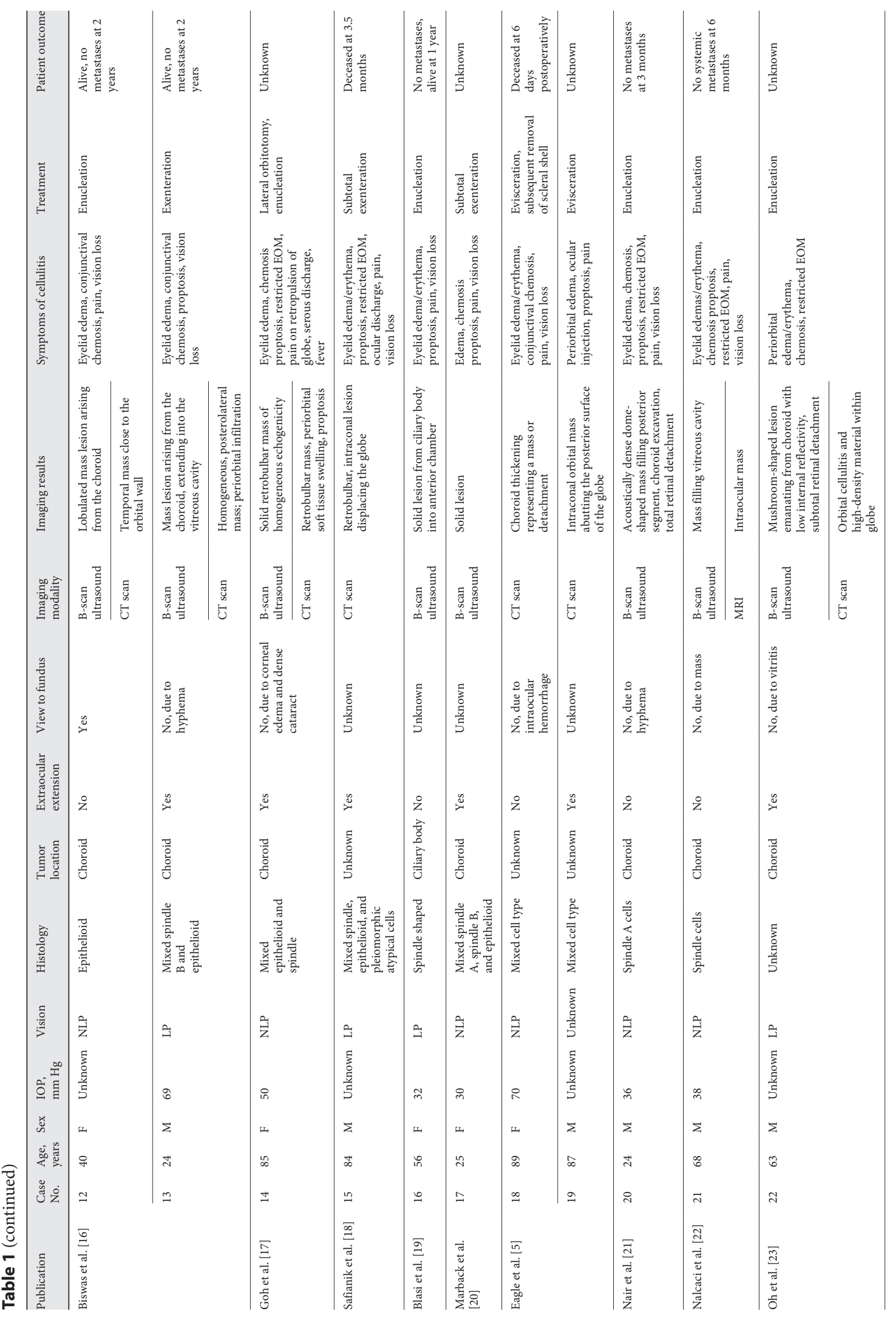




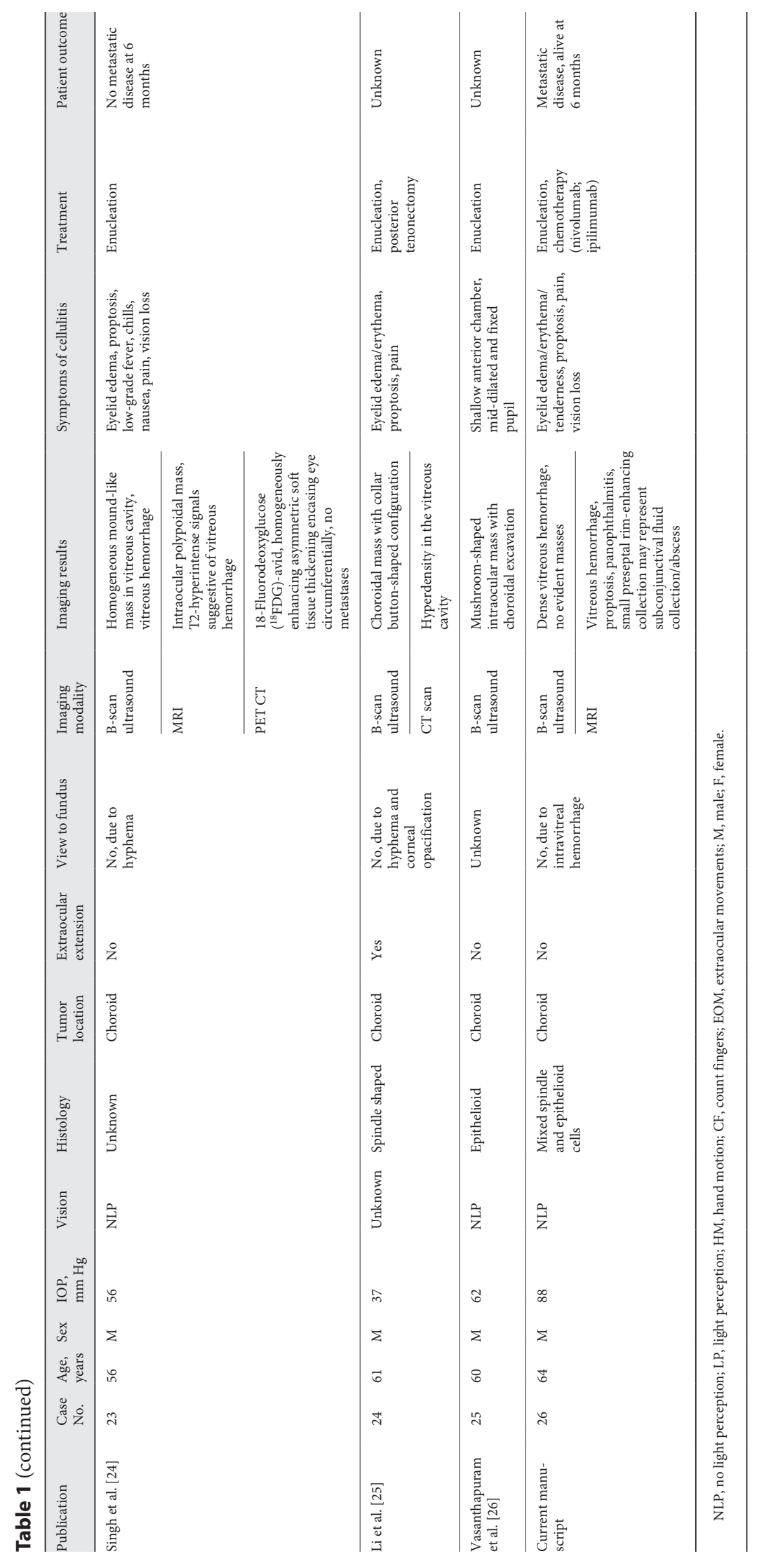


case reports or series, encompassing an additional 25 patients, reporting necrotic uveal melanoma presenting with symptoms mimicking orbital cellulitis [5-26]. The cause for the necrosis is not fully understood; however, it is likely that the orbital inflammation in these patients is caused by an inflammatory process triggered by the tumor necrosis. Through the release of inflammatory mediators and cytokines, such as tumor necrosis factor and IL-6, necrotic tissue is capable of inciting uveitis, episcleritis, and panophthalmitis [24]. Inflammation has been attributed to the cytotoxic effects of released cellular debris after a spontaneous infarction [27].

Several potential mechanisms of tumor autoinfarction have been proposed. Reese et al. [27] proposed that lymphocytic activation of endothelial cells leads to thromboses and vessel occlusion, leading to spontaneous infarction and subsequent necrosis. Others have proposed that the rapidly expanding tumor infarcts as it outgrows its blood supply [13, 21, 23].

A proinflammatory phenotype of uveal melanoma has been described in association with increased HLA class 1 and class 2 expression and increased infiltration by macrophages and lymphocytes [28]. Though increased immune infiltration is a positive prognostic factor in other cancers, it is associated with a negative prognosis in uveal melanoma [28]. Several studies have demonstrated the presence of a lymphocytic infiltrate in a high percentage of necrotic uveal melanomas $[27,29,30]$. Of the reviewed cases, 53.8\% contained epithelioid cells, which are also associated with increased cellular infiltrate and higher HLA class 1 and 2 expression [31,32]. Based on the previously reported association between necrotic uveal melanoma and increased inflammatory infiltrate, it is possible that patients with necrotic uveal melanoma have a predilection towards the inflammatory phenotype, with subsequent vascular damage and autoinfarction [27-32]. Alternatively, posterior segment tumors can induce neovascularization of the iris, possibly also through an inflammatory mechanism $[33,34]$. This can result in necrosis secondary to acute angle closure.

In our case, histopathologic examination revealed extensive necrosis of the iris, ciliary body, and neurosensory retina. There was a reactive inflammatory process to the necrotic mass, with numerous melanin-laden macrophages infiltrating the darkly pigmented mass of necrotic spindle and epithelioid cells. The tumor size could not be determined because the exact transition between the necrotic tumor and the necrotic response to the tumor was unclear. Our case is only the second case where no viable tumor cells were identified on histopathology [10].

\section{Conclusion}

Orbital inflammation is a rare manifestation of uveal melanoma. Autoinfarction and tumor necrosis leads to a proinflammatory state which may lead to orbital inflammation, even in the absence of extrascleral extension of the tumor. Necrotic tumors may not be visible, thus an occult tumor must always be considered and ruled out in patients with severe ocular inflammation where the view to the fundus is obscured.

\section{Statement of Ethics}

This study did not require review by our Institutional Review Board. We report a case of necrotic uveal melanoma presenting with orbital inflammation mimicking orbital cellulitis and present a comprehensive review of the literature and tabulation of reported cases. Written consent was obtained from the patient to publish medical history and clinical photographs.

\section{Conflict of Interest Statement}

The authors have no conflicts of interest to declare.

\section{Funding Sources}

The authors have no funding sources to declare.

\section{Author Contributions}

Ahmad Abdel-Aty prepared the manuscript and conducted the literature review. Wendy L. Linderman contributed to the preparation of the manuscript and in the development of inclusion/exclusion criteria for reviewed articles. Ninani Kombo evaluated clinical data and contributed to the preparation of the manuscript. John Sinard provided interpretation of the histopathology and contributed to the preparation of the manuscript. Renelle PointdujourLim evaluated clinical data and contributed to the preparation of the manuscript.

\section{References}

Ocul Oncol Pathol 2022;8:1-8

1 Kashyap S, Meel R, Singh L, Singh M. Uveal melanoma. Semin Diagn Pathol. 2016;33: 141-7.

2 Rodríguez A, Dueñas-Gonzalez A, DelgadoPelayo S. Clinical presentation and management of uveal melanoma. Mol Clin Oncol. 2016;5(6):675-7.

3 Krantz BA, Dave N, Komatsubara KM, Marr BP, Carvajal RD. Uveal melanoma: epidemiology, etiology, and treatment of primary disease. Clin Ophthalmol. 2017;11:279-89. 
4 Damato EM, Damato BE. Detection and time to treatment of uveal melanoma in the United Kingdom: an evaluation of 2,384 patients. Ophthalmology. 2012;119(8):1582-9.

5 Eagle RC, Grossniklaus HE, Syed N, Hogan RN, Lloyd WC, Folberg R. Inadvertent evisceration of eyes containing uveal melanoma. Arch Ophthalmol. 2009;127(2):141-5.

6 Kline LB, Bright M, Brownstein S. Uveal melanoma presenting as post-traumatic choroidal hemorrhage and panophthalmitis. Can J Ophthalmol. 1977 Jul;12(3):226-9.

7 Fraser DJ, Font RL. Ocular inflammation and hemorrhage as initial manifestations of uveal malignant melanoma: incidence and prognosis. Arch Ophthalmol. 1979;97(7):1311-4.

8 Sassani JW, Weinstein JM, Graham WP. Massively invasive diffuse choroidal melanoma. Arch Ophthalmol. 1985 Jul;103(7):945-8.

9 Margo CE, Pusateri TJ, Ulshafer RJ, Keller RK. Lipid crystals in malignant melanoma of the choroid. Retina. 1990;10(1):68-71.

10 Lea SJ, Livesey SJ, Lowe J, Rothwell I, Haworth SM. Disappearance of ocular malignant melanoma on computerised scan after spontaneous necrosis: clinical, radiological and pathological features. Eye. 1991;5(Pt 6):748-50.

11 Rose GE, Hoh HB, Harrad RA, Hungerford JL. Intraocular malignant melanomas presenting with orbital inflammation. Eye. 1993; 7(Pt 4):539-41.

12 Tabassian A, Zuravleff JJ. Necrotic choroidal melanoma with orbital inflammation. Arch Ophthalmol. 1995 Dec;113(12):1576-7.

13 Mauriello JA, Tello C, Kishore K, Hidayat AA. Carcinoma of the ciliary epithelium presenting as orbital cellulitis. Orbit. 1996;15(1):47-51.

14 Brannan S, Browne B, Clark BJ. Massive infarction of ocular tissues complicating a necrotic uveal melanoma. Eye. 1998;12(Pt 2): $324-5$.
15 Fezza J, Chaudhry IA, Kwon YH, Grannum EE, Sinard J, Wolfley DE. Orbital melanoma presenting as orbital cellulitis: a clinicopathologic report. Ophthalmic Plast Reconstr Surg. 1998;14(4):286-9.

16 Biswas J, Ahuja VK, Shanmugam MP, Kurian R, Fernandez T. Malignant melanoma of the choroid presenting as orbital cellulitis: report of two cases with a review of the literature. Orbit. 1999;18(2):123-30.

17 Goh AS, Francis IC, Kappagoda MB, Filipic M. Orbital inflammation in a patient with extrascleral spread of choroidal malignant melanoma. Clin Exp Ophthalmol. 2001;29(2): 97-9.

18 Safianik B, Vardizer Y, Garzozi HJ. Invasive malignant melanoma of the uvea, presenting with neovascular glaucoma and orbital cellulitis. Ann Ophthalmol. 2002;34(4):222-3.

19 Blasi MA, Giammaria D, Balestrazzi E. Necrotic uveal melanoma with orbital inflammation. Eur J Ophthalmol. 2006;16:647-50.

20 Marback EF, Oliveira RDC, Silva CMP, Andrade IF, Marback RL. Uveal melanoma: documented progression to necrosis with panophthalmitis and extrascleral invasion. Rev Brasileira de oftalmol. 2005;65(3):186-90.

21 Nair AG, Kaliki S, Ali MJ, Naik MN, Vemuganti GK. Intraocular malignant melanoma of the choroid presenting as orbital cellulitis. Int Ophthalmol. 2014;34(3):647-50.

22 Nalcaci S, Palamar M, Yaman B, Akalin T, Mentes J. Choroidal malignant melanoma with no extraocular extension presenting as orbital cellulitis. Orbit. 2016;35(5):285-7.

23 Oh LJ, Dunn H, Cherepanoff S, Giblin M. Orbital cellulitis and secondary angle closure: a rare presentation of choroidal melanoma. Ocul Oncol Pathol. 2018;4(2):130-3.

24 Singh P, Sen S, Banerjee M, Meel R. Choroidal melanoma masquerading as orbital cellulitis. BMJ Case Rep. 2018;11(1).
25 Li AL, Kang JY, Berry DE, Patel KB, Hendrick AM. Necrotic uveal melanoma presenting as orbital cellulitis with intraocular hemorrhage: a case report. Am J Ophthalmol Case Rep. 2019;16:100557.

26 Vasanthapuram VH, Mishra DK, Kaliki S. Choroidal Melanoma With Optic Nerve Infiltration Presenting as Orbital Pseudocellulitis. Ophthalmic Plast Reconstr Surg. 2019 Jul; 135(4):e106.

27 Reese AB, Archila EA, Jones IS, Cooper WC. Necrosis of malignant melanoma of the choroid. Am J Ophthalmol. 1970;69(1):91-104.

28 Jager MJ, Ly LV, El Filali M, Madigan MC. Macrophages in uveal melanoma and in experimental ocular tumor models: friends or foes? Prog Retin Eye Res. 2011;30(2):129-46.

29 Bujara K. Necrotic malignant melanomas of the choroid and ciliary body. A clinicopathological and statistical study. Graefes Arch Clin Exp Ophthalmol. 1982;219(1):40-3.

30 Lang JR, Davidorf FH, Baba N. The prognostic significance of lymphocytic infiltration in malignant melanoma of the choroid. Cancer. 1977;40(5):2388-94.

31 Rossi E, Schinzari G, Zizzari IG, Maiorano BA, Pagliara MM, Sammarco MG, et al. Immunological backbone of uveal melanoma: is there a rationale for immunotherapy? Cancers. 2019;11(8):1055.

32 Bronkhorst IH, Jager MJ. Uveal melanoma: the inflammatory microenvironment. J Innate Immun. 2012;4(5-6):454-62. http:// dx.doi.org/10.1159/000334576.

33 Camp DA, Yadav P, Dalvin LA, Shields CL. Glaucoma secondary to intraocular tumors: mechanisms and management. Curr Opin Ophthalmol. 2019;30(2):71-81.

34 Chan CC, Nussenblatt RB. Ocular inflammation and neovascularization. In: Ocular angiogenesis: Humana Press; 2006. p. 291-305. 